\title{
A Study on the Rate of Convergence of Chlodovsky-Durrmeyer Operator and Their Bézier Variant
}

\author{
S. K. Tiwari ${ }^{1}$, V.K. Gupta ${ }^{2}$, Yogita Parmar ${ }^{3}$ \\ ${ }^{1:}$ Department of Mathematics, School of Studies in mathematics, Vikram University, Ujjain (M.P.) \\ 2: Department of Mathematics, Govt. Madhav Science College Ujjain (M.P.) \\ .3: Preseach Scholar, School of Studies in mathematics, Vikram University, Ujjain (M.P.)
}

\begin{abstract}
In this paper, we have studied the Bézier variant of Chlodovsky-Durrmeyer operators $D_{m, \vartheta}$ for function $f$ measurable and locally bounded on the interval $[0, \infty)$. In this we improved the result given by Ibikl $E$. And Karsli H. [14]. We estimate the rate of pointwise convergence of $\left(D_{m, \vartheta} f\right)(x)$ at those $x>0$ at which the one-sided limits $f(x+), f(x-)$ exist by using the Chanturia modulus of variation. In the special case $\vartheta=1$ the recent result of Ibikl E. And Karsli $H$. [14] concerning the Chlodowsky-Durrmeyer operators $D_{m}$ is essentially improved and extended to more general classes of functions.
\end{abstract}

Keywords: Rate of convergence, Chlodovsky-Durrmeyer operator, Bézier basis, Chanturia modulus of variation, $p$-th power variation.

\section{Introduction}

For a function the classical Bernstein-Durrmeyer operators (see [7]) $M_{n}$ applied to $f$ are define as

$$
\left(M_{m} f\right)(x)=(m+1) \sum_{i=0}^{m} p_{m, i}(x) \int_{0}^{1} f(t) p_{m, i}(t) d t, \quad x \in[0,1]
$$

where $p_{m, i}(x)=\left(\begin{array}{c}m \\ i\end{array}\right) x^{i}(1-x)^{m-i}$.

Several researchers have studied approximation properties of the operators $M_{n}$ ([8], [10]) for function of bounded variation defined on the interval $[0,1]$. After that Zeng and Chen [22] defined the Bézier variant of Durrmeyer operators as

$$
\left(M_{m, \vartheta} f\right)(x)=(m+1) \sum_{i=0}^{m} Q_{m, i}^{(\vartheta)}(x) \int_{0}^{1} f(t) p_{m, i}(t) d t,
$$

where $Q_{m, i}^{(\vartheta)}(x)=J_{m, i}^{\vartheta}(x)-J_{m, i+1}^{\vartheta}(x)$ and $J_{m, i}(x)=\sum_{j=i}^{m} p_{m, j}(x)$ for $i=0,1,2, \ldots, m$,

$J_{m, m+i}(x)=0$ are the Bézier basis function which is introduced by P. Bézier [4] and estimated the rate of convergence of $M_{m, \vartheta} f$ for functions of bounded variation on the interval [0,1].

Let $\mathrm{X}_{l o c}[0, \infty)$ be the class of all complex valued function measurable and locally bounded on the interval $[0, \infty)$. For $f \in X_{\text {loc }}[0, \infty)$ the Chlodowsky-Durrmeyer operator $D_{m}$ are defined as

$$
\left(D_{m} f\right)(x)=\frac{m+1}{a_{m}} \sum_{i=0}^{m} P_{m, i}\left(\frac{x}{a_{m}}\right) \int_{0}^{a_{m}} f(t) P_{m, i}\left(\frac{t}{a_{m}}\right) d t, \quad 0 \leq x \leq a_{m} .
$$

where $\left(a_{m}\right)$ is a positive increasing sequence with the properties

$$
\lim _{m \rightarrow \infty} a_{m}=\infty \text { and } \lim _{m \rightarrow \infty} \frac{a_{m}}{m}=0
$$

For $f \in X_{\text {loc }}[0, \infty)$ and $\vartheta \geq 1$, we introduce the Bézier variant of Chlodowsky-Durrmeyer operators $D_{m, \vartheta}$ as follows

$$
\left(D_{m, \vartheta} f\right)(x)=\frac{m+1}{a_{m}} \sum_{i=0}^{m} Q_{m, i}^{(\vartheta)}\left(\frac{x}{a_{m}}\right) \int_{0}^{a_{m}} f(t) P_{m, i}\left(\frac{t}{a_{m}}\right) d t, \quad 0 \leq x \leq a_{m} .
$$

Obviously, $D_{m, \vartheta}$ is a positive linear operator and $\left(D_{m, \vartheta} 1\right)(x)=1$. In particular, when $\vartheta=1$ the operators (5) reduce to operators (3).

Recently Agratini [1], Aniol and Pych-Taberska [3], Pych-Taberska [20], and Gupta [11, 12] have investigated the rate of pointwise convergence for Kantorovich and Durrmeyer Type Baskakov-Bézier and Bézier operators using a different approach. They have proved their theorems in terms of the Chanturia modulus of variation, which is a generalization of the classical Jordan variation. It is useful to point out that a deeper analysis of the Chanturia modulus of variation can be found in [6], but actually the modulus of variation was introduced for the first time by Langrange [18]. Although the Chanturia modulus of variation was defined as a 
generalization of the classical variation nearly four decades years ago, it was not used to a sufficient extent to solve the problem mentioned above.

The paper is concerned with the rate of pointwise convergence of the operators (5) when $f$ belong to $X_{l o c}[0, \infty)$. Using the Chanturia modulus of variation defined in [6], we examine the rate of pointwise convergence of $\left(D_{m, \vartheta} f\right)(x)$ at the points of continuity and at the first kind discontinuity points of $f$.

For some important papers on different operators related to the present study we refer the readers to Gupta et. Al. [9, 21] and Zeng and Piriou [23]. It is necessary to point out that in the present paper we extend and improve the result of Ibikli E. and Karsli H.[14] for Chlodowsky-Durrmeyer operators.

We being by giving

Definition 1.1 Let $f$ be a bounded function on a compact interval $I=[a, b]$. The modulus of variation $\mu_{m}(f ;[a, b])$ of a function $f$ is defined for nonnegative integers $m$ as

$$
\mu_{0}(f ;[a, b])=0
$$

and for $m \geq 1$ as

$$
\mu_{m}(f ;[a, b])=\sup _{\pi_{m}} \sum_{i=0}^{m-1}\left|f\left(x_{2 i+1}\right)-f\left(x_{2 i}\right)\right|,
$$

where $\pi_{m}$ is an arbitrary system of $m$ disjoint intervals $\left(x_{2 i}, x_{2 i+1}\right), i=0,1, \ldots, m-1$, i.e., $a \leq x_{0}<x_{1} \leq x_{2}<x_{3} \leq \cdots \leq x_{2 m-2}<x_{2 m-1} \leq b$.

The modulus of variation of any function is a non-decreasing function of $m$. Some other properties of this modulus can be found in [6].

If $f \in B V_{p}(I), p \geq 1$, i.e., if $f$ of $p$-th bounded power variation on $I$, then for every $i \in \mathbb{N}$,

$$
\mu_{i}(f ; I) \leq i^{1-1 / p} V_{p}(f, I),
$$

where $V_{p}(f, I)$ denotes the total $p$-th bounded power variation of $f$ on $I$, defined as the upper bound of the set of numbers $\left(\sum_{j}\left|f\left(i_{j}\right)-f\left(l_{j}\right)\right|^{p}\right)^{1 / p}$ over all finite systems of non-overlapping intervals $\left(i_{j}, l_{j}\right) \subset I$.

We also consider the class $B V_{l o c}^{p}[0, \infty), p \geq 1$, consisting of all function of bounded $p$-th power variation on every compact interval $I \subset[0, \infty)$.

In the sequel it will be always assumed that the sequence $\left(a_{m}\right)$ satisfies the fundamental conditions (4). The symbol $[a]$ will be denote the greatest integer not greater than $a$.

Remark. Now, let us consider the special case $\vartheta=1, p=1$, and let us suppose that function $f$ is of bounded variation in the Jorden sense on the whole interval $[0, \infty)$

$(f \in B V[0, \infty))$. Then, for all integers $m$ such that $a_{m}>2 x$ and $4 a_{m} \leq m$, we have the following estimation for the rate of convergence of the Chlodowsky-Durrmeyer operators (3):

$$
\begin{gathered}
\left|\left(D_{m, \vartheta} f\right)(x)-\frac{f(x+)+f(x-) \mid \leq 2 V\left(g_{x} ; H_{x}\left(x \sqrt{a_{m} / m}\right)\right.}{2}\right| \leq{ }^{10} a_{m} \\
+\frac{2^{2}}{m x^{2}}\left(x\left(1-\frac{x}{a_{m}}\right)+\frac{a_{m}}{m}\right) \sum_{i=1}^{2\left[m / a_{m}\right]} V\left(g_{x} ; H_{x}\left(\frac{x}{\sqrt{i}}\right)\right) \\
+\frac{4 M a_{m}}{m x^{2}}\left(x\left(1-\frac{x}{a_{m}}\right)+\frac{a_{m}}{m}\right)+\frac{2|f(x+)-f(x-)|}{\sqrt{\frac{m x}{a_{m}}\left(1-\frac{x}{a_{m}}\right)}}
\end{gathered}
$$

where $M=\operatorname{Sup}_{0 \leq x<\infty}|f(x)|$ and $V\left(g_{x} ; H\right)$ denotes the Jordan variation of $g_{x}$ on the interval H.

The above estimation is essentially better than the estimation presented in [14]. Namely, it is easy to see that the right-hand side of the main inequality given in Theorem 1.1 in [14] is not convergent to zero for all function $f \in B V[0, \infty)$ and for all sequences $\left(a_{m}\right)$ satisfying $(4)$.

\section{Auxilary Result}

In this section we give certain results, which are necessary to prove the main result.

For this, let us introduce the following notation. Given any $x \in\left[0, a_{m}\right]$ and any non-negative integer $q$, we write

$$
\begin{gathered}
\psi_{x}^{q}(t):=(t-x)^{q} \text { for } t \in[0, \infty), \\
W_{m, q}(x):=\left(D_{m} \psi_{x}^{q}\right)(x) \equiv \frac{m+1}{a_{m}} \sum_{i=0}^{m} P_{m, i}\left(\frac{x}{a_{m}}\right) \int_{0}^{a_{m}}(t-x)^{q} P_{m, i}\left(\frac{t}{a_{m}}\right) d t .
\end{gathered}
$$

Lemma 2.1 If $m \in \mathbb{N}, x \in\left[0, a_{m}\right]$, then

$$
W_{m, 0}(x)=1, \quad W_{m, 1}(x)=\frac{a_{m}-2 x}{m+2},
$$


and, for $\mathrm{q}>1$,

$$
W_{m, 2}(x)=\frac{2(m-3)\left(a_{m}-x\right) x}{(m+2)(m+3)}+\frac{2 a_{m}^{2}}{(m+2)(m+3)}
$$

$$
W_{m, 2 q}(x)=\left(\frac{a_{m}}{m}\right)^{q} \sum_{j=0}^{q} \beta_{j, q}\left(x\left(1-\frac{x}{a_{m}}\right)\right)^{q-j}\left(\frac{a_{m}}{m}\right)^{j},
$$

where $\beta_{j, q}$ are real numbers independent of $x$ and bounded uniformly in $m$. Moreover, for $m \geq 2$

and, for $\mathrm{q}>1$,

$$
W_{m, 2 q}(x) \leq 2 \frac{a_{m}}{m}\left(x\left(1-\frac{x}{a_{m}}\right)+\frac{a_{m}}{m}\right)
$$

$$
W_{m, 2 q}(x) \leq c_{q}\left(\frac{a_{m}}{m}\right)^{q}\left(x\left(1-\frac{x}{a_{m}}\right)+\frac{a_{m}}{m}\right)^{q},
$$

where $c_{q}$ is a positive constant depending only on $q$.

Proof. Formulas for $W_{m, 0}, W_{m, 1}, W_{m, 2}$ and inequality (9) follow by simple calculation. Suppose $q>1$ and put $y:=x / a_{m}$. Then $y \in[0,1]$ and

$$
\begin{array}{r}
W_{m, 2 q}(x)=\frac{m+1}{a_{m}} \sum_{i=0}^{m} P_{m, i}(y) \int_{0}^{a_{m}}\left(t-y a_{m}\right)^{2 q} P_{m, i}\left(\frac{t}{a_{m}}\right) d t \\
=(m+1) a_{m}^{2 q} \sum_{i=0}^{m} P_{m, i}(y) \int_{0}^{1}(s-y)^{2 q} P_{m, i}(s) d s=a_{m}^{2 q}\left(M_{m} \psi_{y}^{2 q}\right)(y),
\end{array}
$$

where $M_{m}$ is the classical Bernstein-Durrmeyer operator (1).

The representation formula (8) follows at once from the known identity

$$
\left(M_{m} \psi_{y}^{2 q}\right)(y)=\sum_{j=0}^{q} \beta_{j, q, m}\left(\frac{y(1-y)}{m}\right)^{q-1} m^{-2 j},
$$

where $\beta_{j, q, m}$ are real numbers independent of $y$ and bounded uniformly in $m$ (see [13], Lemma 4.8 with $c=$ $-1)$. Now, let us observe that for $y \in[0,1 / m] y \in\left[1-\frac{1}{m}, 1\right], m \geq 2$, one has $y(1-y) \leq \frac{m-1}{m^{2}}$ and

$$
\left(M_{m} \psi_{y}^{2 q}\right)(y)=\sum_{j=0}^{q}\left|\beta_{j, q, m}\right|\left(\frac{m-1}{m^{3}}\right)^{q-1} m^{-2 j} \leq \sum_{j=0}^{q} \eta_{j, q} m^{-2 q},
$$

where $\eta_{j, q}$ are non-negative numbers depending only on $j$ and $q$. If $y \in\left[\frac{1}{m}, 1-\frac{1}{m}\right]$ then $\frac{1}{m y(1-y)} \leq \frac{m}{m-1} \leq 2$ and

Consequently,

$$
\begin{aligned}
\left(M_{m} \psi_{y}^{2 q}\right)(y) & =\left(\frac{y(1-y)}{m}\right)^{q} \sum_{j=0}^{q}\left|\beta_{j, q, m}\right| \frac{1}{(m y(1-y))^{j}}, \\
& \leq\left(\frac{y(1-y)}{m}\right)^{q} \sum_{j=0}^{q} \eta_{j, q} 2^{j} .
\end{aligned}
$$

$$
\left(M_{m} \psi_{y}^{2 q}\right)(y) \leq \frac{C_{q}}{m^{q}}\left(y(1-y)+\frac{1}{m}\right)^{q} \text { with } C_{q}=\sum_{j=0}^{q} \eta_{j, q} 2^{j} .
$$

Taking in (11) and in the above inequality $y=x / a_{m}$ we easily obtain estimation (10).

Lemma 2.2 Let $x \in(0, \infty)$ and let

Then

$$
K_{m, \vartheta}\left(\frac{x}{a_{m}}, \frac{t}{a_{m}}\right):=\frac{m+1}{a_{m}} \sum_{i=0}^{m} Q_{m, i}^{(\vartheta)}\left(\frac{x}{a_{m}}\right) P_{m, i}\left(\frac{t}{a_{m}}\right) .
$$

and

$$
\int_{t}^{a_{m}} K_{m, \vartheta}\left(\frac{x}{a_{m}}, \frac{u}{a_{m}}\right) d u \leq \frac{\vartheta}{(t-x)^{2}} W_{m, 2}(x) \text { if } x<t
$$

$$
\int_{0}^{t} K_{m, \vartheta}\left(\frac{x}{a_{m}}, \frac{u}{a_{m}}\right) d u \leq \frac{\vartheta}{(x-t)^{2}} W_{m, 2}(x) \text { if } 0<t<x,
$$


where $W_{m, 2}(x)$ is given by (7) (with $q=2$ ).

Proof. As is known $Q_{m, i}^{(\vartheta)}(x) \leq \vartheta P_{m, i}(x)$ for $\vartheta \geq 1$. Hence, if $x<t$, then

$$
\begin{gathered}
\int_{t}^{a_{m}} K_{m, \vartheta}\left(\frac{x}{a_{m}}, \frac{u}{a_{m}}\right) d u \leq \frac{1}{(t-x)^{2}} \int_{t}^{a_{m}}(u-x)^{2} K_{m, \vartheta}\left(\frac{x}{a_{m}}, \frac{u}{a_{m}}\right) d u \\
\leq \frac{1}{(t-x)^{2}}\left(D_{m, \vartheta} \psi_{x}^{2}\right)(x) \leq \frac{\vartheta}{(t-x)^{2}}\left(D_{m} \psi_{x}^{2}\right)(x)=\frac{\vartheta}{(t-x)^{2}} W_{m, 2}(x) .
\end{gathered}
$$

The proof of (13) is similar.

In order to formulate the next lemma we introduce the following intervals. If $x>0$, we write

$$
I_{x}(u):=[x+u, x] \cap[0, \infty) \text { if } u<0
$$

$$
I_{x}(u):=[x, x+u] \text { if } u>0
$$

Lemma 2.3 Let $f \in X_{\text {loc }}[0, \infty)$ and let the one-sided limits $f(x+), f(x-)$ exist at a fixed point $x \in(0, \infty)$. Consider the function $g_{x}$ defined by (6) and write $d_{m}:=\sqrt{a_{m} / m}$. If $h=-x$ or $h=x$, then for all integers $m$ such that $d_{m} \leq 1 / 2$ we have

$$
\begin{aligned}
& \left|\int_{I_{x}(h)} g_{x}(t) K_{m, \vartheta}\left(\frac{x}{a_{m}}, \frac{t}{a_{m}}\right) d t\right| \leq v_{1}\left(g_{x} ; I_{x}\left(h d_{m}\right)\right) \\
& +\frac{8 \vartheta W_{m, 2}(x)}{h^{2} d_{m}^{2}}\left[\sum_{j=1}^{n-1} \frac{v_{j}\left(g_{x} ; I_{x}\left(j h d_{m}\right)\right)}{j^{3}}+\frac{v_{n}\left(g_{x} ; I_{x}(h)\right)}{n^{2}}\right],
\end{aligned}
$$

where $n=\left[1 / d_{m}\right]$ and $W_{m, 2}(x)$ is estimated in (9).

Proof. Restricting the proof to $h=-x$ we define the point $t_{j}=x+j h d_{n}$ for $j=1,2,3, \ldots, n+1$ and we denote $t_{n+1}=0$. Put $T_{j}=\left[t_{j}, x\right]$ for $j=1,2,3, \ldots, n+1$ and we have

$$
\begin{gathered}
\int_{I_{x}(h)} g_{x}(t) K_{m, \vartheta}\left(\frac{x}{a_{m}}, \frac{t}{a_{m}}\right) d t \leq \int_{t_{1}}^{x} g_{x}(t) K_{m, \vartheta}\left(\frac{x}{a_{m}}, \frac{t}{a_{m}}\right) d t \\
+\sum_{j=1}^{n} g_{x}\left(t_{j}\right) \int_{t_{j+1}}^{t_{j}} K_{m, \vartheta}\left(\frac{x}{t_{m}}, \frac{t}{a_{m}}\right) d t+\sum_{j=1}^{n} \int_{t_{j+1}}^{n}\left(g_{x}(t)-g_{x}\left(t_{j}\right)\right) K_{m, \vartheta}\left(\frac{x}{a_{m}}, \frac{t}{a_{m}}\right) d t \\
=I_{1}(m, x)+I_{2}(m, x)+I_{3}(m, x), \quad \text { say }
\end{gathered}
$$

Clearly,

$$
\begin{aligned}
\left|I_{1}(m, x)\right| & \leq \int_{t_{1}}^{x}\left|\left(g_{x}(t)-g_{x}(x)\right)\right| K_{m, \vartheta}\left(\frac{x}{a_{m}}, \frac{t}{a_{m}}\right) d t \\
& \leq v_{1}\left(g_{x} ; T_{1}\right) \int_{0}^{a_{m}} K_{m, \vartheta}\left(\frac{x}{a_{m}}, \frac{t}{a_{m}}\right) d t=v_{1}\left(g_{x} ; T_{1}\right) .
\end{aligned}
$$

By the Abel lemma on summation by parts and by (13) we have

$$
\begin{aligned}
\left|I_{2}(m, x)\right| & \leq\left|g_{x}\left(t_{1}\right)\right| \int_{0}^{t_{1}} K_{m, \vartheta}\left(\frac{x}{a_{m}}, \frac{t}{a_{m}}\right) d t+\sum_{j=1}^{n-1}\left|g_{x}\left(t_{j+1}\right)-g_{x}\left(t_{j}\right)\right| \int_{0}^{t_{j+1}} K_{m, \vartheta}\left(\frac{x}{a_{m}}, \frac{t}{a_{m}}\right) d t \\
& \leq \frac{\vartheta W_{m, 2}(x)}{h^{2} d_{m}^{2}}\left(\left|g_{x}\left(t_{1}\right)-g_{x}(x)\right|+\sum_{j=1}^{n-1}\left|g_{x}\left(t_{j+1}\right)-g_{x}\left(t_{j}\right)\right| \frac{1}{(j+1)^{2}}\right) \\
= & \frac{\vartheta W_{m, 2}(x)}{h^{2} d_{m}^{2}}\left(\left|g_{x}\left(t_{1}\right)-g_{x}(x)\right|+\sum_{j=1}^{n-2} \sum_{i=1}^{j}\left|g_{x}\left(t_{i+1}\right)-g_{x}\left(t_{i}\right)\right|\left(\frac{1}{(j+1)^{2}}-\frac{1}{(j+2)^{2}}\right)\right. \\
\left.\quad+\frac{1}{n^{2}} \sum_{i=1}^{n}\left|g_{x}\left(t_{i+1}\right)-g_{x}\left(t_{i}\right)\right|\right) & \\
\leq & \frac{\vartheta W_{m, 2}(x)}{h^{2} d_{m}^{2}}\left(v_{1}\left(g_{x} ; T_{1}\right)+2 \sum_{j=1}^{n-2} \frac{v_{j+1}\left(g_{x} ; T_{j+1}\right)}{(j+1)^{3}}+\frac{v_{n}\left(g_{x} ; T_{n}\right)}{n^{2}}\right)
\end{aligned}
$$




$$
\leq \frac{\vartheta W_{m, 2}(x)}{h^{2} d_{m}^{2}}\left(2 \sum_{j=1}^{n-1} \frac{v_{j}\left(g_{x} ; T_{j}\right)}{j^{3}}+\frac{v_{n}\left(g_{x} ; T_{n+1}\right)}{n^{2}}\right) .
$$

Next, in view of (13) and the Abel transformation,

$$
\begin{aligned}
&\left|I_{3}(m, x)\right| \leq \sum_{j=1}^{n} v_{1}\left(g_{x} ;\left[t_{j+1}, t_{j}\right]\right) \int_{t_{j+1}}^{t_{1}} K_{m, \vartheta}\left(\frac{x}{a_{m}}, \frac{t}{a_{m}}\right) d t \\
& \leq \frac{\vartheta W_{m, 2}(x)}{h^{2} d_{m}^{2}} \sum_{j=1}^{n} \frac{v_{1}\left(g_{x} ;\left[t_{j+1}, t_{j}\right]\right)}{j^{2}} \\
&=\frac{\vartheta W_{m, 2}(x)}{h^{2} d_{m}^{2}}\left(\sum_{i=1}^{n} \frac{v_{1}\left(g_{x} ;\left[t_{i+1}, t_{i}\right]\right)}{n^{2}}+\sum_{j=1}^{n-1} \sum_{i=1}^{j} v_{1}\left(g_{x} ;\left[t_{i+1}, t_{i}\right]\right)\left(\frac{1}{j^{2}}-\frac{1}{(j+1)^{2}}\right)\right) \\
& \leq \\
& \leq \frac{\vartheta W_{m, 2}(x)}{h^{2} d_{m}^{2}}\left(\frac{v_{n}\left(g_{x} ; T_{n+1}\right)}{n^{2}}+6 \sum_{j=1}^{n-1} \frac{v_{j}\left(g_{x} ; T_{j+1}\right)}{(j+1)^{3}}\right) \\
& \leq \frac{\vartheta W_{m, 2}(x)}{h^{2} d_{m}^{2}}\left(\frac{v_{n}\left(g_{x} ; T_{n+1}\right)}{n^{2}}+6 \sum_{j=2}^{n} \frac{v_{j}\left(g_{x} ; T_{j}\right)}{j^{3}}\right)
\end{aligned}
$$

Combining the result and observing that $T_{j}=I_{x}\left(j h d_{m}\right)$ we get the desired estimation for $h=-x$. In the case of $h$ $=x$ the proof runs analogously; we use inequality (12) instead of (13).

In this section we prove our main theorems.

\section{Main Results}

Theorem 3.1 Let $f \in X_{\text {loc }}[0, \infty)$ and let the one-sided limits $f(x+), f(x-)$ exist at a fixed point $x \in(0, \infty)$. Then, for all integers $m$ such that $a_{m}>2 x$ and $4 a_{m} \leq m$ one has

$$
\begin{gathered}
\left|\left(D_{m, \vartheta} f\right)(x)-\frac{f(x+)+\vartheta f(x-)}{\vartheta+1}\right| \leq 2 \mu_{1}\left(g_{x} ; H_{x}\left(x \sqrt{a_{m} / m}\right)\right. \\
+\frac{32 \vartheta}{x^{2}}\left(x\left(1-\frac{x}{a_{m}}\right)+\frac{a_{m}}{m}\right)\left[\sum_{j=1}^{n-1} \frac{\mu_{j}\left(g_{x} ; H_{x}\left(j x \sqrt{a_{m} / m}\right)\right.}{j^{3}}+\frac{\mu_{n}\left(g_{x} ; H_{x}(x)\right)}{n^{2}}\right] \\
+\frac{2 \vartheta C_{q}}{x^{2 q}} \varphi\left(a_{m} ; f\right)\left(\frac{a_{m}}{m}\right)^{q}\left(x\left(1-\frac{x}{a_{m}}\right)+\frac{a_{m}}{m}\right)^{q}+\frac{2 \vartheta|f(x+)-f(x-)|}{\sqrt{\frac{m x}{a_{m}}\left(1-\frac{x}{a_{m}}\right)}},
\end{gathered}
$$

where $n=\left[\sqrt{m / a_{m}}\right], H_{x}(u)=[x-u, x+u]$ for $0 \leq u \leq x, \varphi(a ; f)=\sup _{0 \leq t \leq b}|f(t)|$

$$
g_{x}(t)= \begin{cases}f(t)-f(x+) & \text { if } t>x, \\ 0 & \text { if } t=x, \\ f(t)-f(x-) & \text { if } 0 \leq t<x,\end{cases}
$$

$q$ is an arbitrary positive integer and $c_{q}$ is a positive constant depending only on $q$.

Proof. We decompose $f(t)$ into four parts as

$$
\begin{gathered}
f(t)=\frac{f(x+)+\vartheta f(x-)}{\vartheta+1}+\frac{f(x+)-f(x-)}{2}\left[\operatorname{sgn}_{x}(t)+\frac{\vartheta-1}{\vartheta+1}\right]+g_{x}(t) \\
+\delta_{x}(t)\left[f(x)+\frac{f(x+)-f(x-)}{2}\right]
\end{gathered}
$$

where $g_{x}(t)$ is defined as (14) and $s g n_{x}(t):=\operatorname{sgn}(t-x)$,

From (15) we have

$$
\delta_{x}(t)= \begin{cases}1, & x=t, \\ 0, & x \neq t,\end{cases}
$$

$$
\begin{aligned}
& \left(D_{m, \vartheta} f\right)(x)=\frac{f(x+)+\vartheta f(x-)}{\vartheta+1}+\left(D_{m, \vartheta} g_{x}\right)(x)+\frac{f(x+)-f(x-)}{2} \\
\times & {\left[\left(D_{m, \vartheta} s g n_{x}\right)(x)+\frac{\vartheta-1}{\vartheta+1}\right]+\left[f(x)-\frac{f(x+)-f(x-)}{2}\right]\left(D_{m, \vartheta} \delta_{x}\right)(x) . }
\end{aligned}
$$

For operators $D_{m, \vartheta}$ using (16) we can observe that the last term on the right hand side vanishes. In addition it is obvious that $\left(D_{m, \vartheta} 1\right)(x)=1$. Hence we have 


$$
\begin{aligned}
& \left|\left(D_{m, \vartheta} f\right)(x)-\frac{f(x+)+\vartheta f(x-)}{\vartheta+1}\right| \\
& \quad \leq\left|\left(D_{m, \vartheta} g_{x}\right)(x)\right|+\left|\frac{f(x+)-f(x-)}{2}\right|\left|\left(D_{m, \vartheta} s g n_{x}\right)(x)+\frac{\vartheta-1}{\vartheta+1}\right|,
\end{aligned}
$$

In order to prove our theorem we need the estimates for $\left(D_{m, \vartheta} g_{x}\right)(x)$ and $\left(D_{m, \vartheta} s g n_{x}\right)(x)+\frac{\vartheta-1}{\vartheta+1}$. follows:

To estimate $\left(D_{m, \vartheta} g_{x}\right)(x)$ with the help of the fixed points $x$ and $2 x$, we decompose it into three parts as

$$
\begin{gathered}
\left|\int_{0}^{a_{m}} g_{x}(t) K_{m, \vartheta}\left(\frac{x}{a_{m}}, \frac{t}{a_{m}}\right) d t\right| \leq\left|\int_{0}^{x} g_{x}(t) K_{m, \vartheta}\left(\frac{x}{a_{m}}, \frac{t}{a_{m}}\right) d t\right| \\
+\left|\int_{x}^{2 x} g_{x}(t) K_{m, \vartheta}\left(\frac{x}{a_{m}}, \frac{t}{a_{m}}\right) d t\right|+\left|\int_{2 x}^{a_{m}} g_{x}(t) K_{m, \vartheta}\left(\frac{x}{a_{m}}, \frac{t}{a_{m}}\right) d t\right| \\
=\left|E_{1, \vartheta}(m, x)\right|+\left|E_{2, \vartheta}(m, x)\right|+\left|E_{3, \vartheta}(m, x)\right|,
\end{gathered}
$$

where $K_{m, \vartheta}\left(\frac{x}{a_{m}}, \frac{t}{a_{m}}\right)$ is defined in Lemma 2.2.

The estimations for $\left|E_{1, \vartheta}(m, x)\right|$ and $\left|E_{2, \vartheta}(m, x)\right|$ are given in Lemma 2.3 in which we put $h=-x$ and $h=x$, respectively. Using the obvious inequality

$$
\square_{j}\left(g_{x} ; I_{x}(-u)\right)+\square_{j}\left(g_{x} ; I_{x}(u)\right) \leq 2 Q_{j}\left(g_{x} ; H_{x}(u)\right),
$$

where $H_{x}(u)=[x-u, x+u], \quad 0<u \leq x$, we obtain

$$
\begin{array}{r}
\left|E_{1, \vartheta}(m, x)\right|+\left|E_{2, \vartheta}(m, x)\right| \leq 2 Q_{j}\left(g_{x} ; H_{x}\left(x \sqrt{a_{m} / m}\right)\right) \\
+\frac{16 \vartheta W_{m, 2}(x) m}{h^{2} d_{m}}\left[\sum_{j=1}^{n-1} \frac{Q_{j}\left(g_{x} ; H_{x}\left(j x \sqrt{a_{m} / m}\right)\right)}{j^{3}}+\frac{a_{n}\left(g_{x} ; H_{x}(x)\right)}{n^{2}}\right] .
\end{array}
$$

Now, we estimate $\left|E_{3, \vartheta}(m, x)\right|$ Clearly, given any $q \in \mathbb{N}$, we have

$$
\begin{aligned}
\left|E_{3, \vartheta}(m, x)\right| & \leq 2 \varphi\left(a_{m} ; f\right) \frac{m+1}{a_{m}} \sum_{i=0}^{m} Q_{m, i}^{(\vartheta)}\left(\frac{x}{a_{m}}\right) \int_{2 x}^{a_{m}} P_{m, i}\left(\frac{t}{a_{m}}\right) d t \\
& \leq 2 \varphi\left(a_{m} ; f\right) \frac{m+1}{x^{2 q} a_{m}} \sum_{i=0}^{m} Q_{m, i}^{(\vartheta)}\left(\frac{x}{a_{m}}\right) \int_{2 x}^{a_{m}}(t-x)^{2 q} P_{m, i}\left(\frac{t}{a_{m}}\right) d t \\
& \leq \frac{2 \vartheta \varphi\left(a_{m} ; f\right)}{x^{2 q}} \frac{m+1}{a_{m}} \sum_{i=0}^{m} P_{m, i}\left(\frac{x}{a_{m}}\right) \int_{0}^{a_{m}}(t-x)^{2 q} P_{m, i}\left(\frac{t}{a_{m}}\right) d t \\
& =\frac{2 \vartheta \varphi\left(a_{m} ; f\right)}{x^{2 q}} W_{m, 2 q}(x) .
\end{aligned}
$$

Finally, replacing $x$ by $x / a_{m}$ in the result of X. M. Zeng and W. Chen [22] (sect. 3, pp. 9-11) we immediately get

$$
\left|\left(D_{m, \vartheta} \operatorname{sgn}_{x}\right)(x)+\frac{\vartheta-1}{\vartheta+1}\right| \leq \frac{4 \vartheta}{\sqrt{m \frac{x}{a_{m}}\left(1-\frac{x}{a_{m}}\right)}}
$$
complete.

Putting (18), (19), (20) and (21) into (17), we get the required result. Thus the proof of Theorem 1 is From Theorem 3.1 and inequality (6) we get

Theorem 3.2 Let $f \in B V_{l o c}^{p}[0, \infty), p \geq 1$ and let $x \in(0, \infty)$. Then, for all integers $m$ such that $a_{m}>2 x$ and $4 a_{m} \leq m$ we have

$$
\begin{gathered}
\left|\left(D_{m, \vartheta} f\right)(x)-\frac{f(x+)+\vartheta f(x-)}{\vartheta+1}\right| \leq 2 V_{p}\left(g_{x} ; H_{x}\left(x \sqrt{a_{m} / m}\right)\right. \\
+\frac{2^{7+1 / p} \vartheta}{x^{2} n^{1+1 / p}}\left(x\left(1-\frac{x}{a_{m}}\right)+\frac{a_{m}}{m}\right) \sum_{i=1}^{(n+1)^{2}-1} \frac{V_{p}\left(g_{x} ; H_{x}\left(\frac{x}{\sqrt{i}}\right)\right)}{(\sqrt{i})^{2-1 / p}}
\end{gathered}
$$




$$
+\frac{2 \vartheta C_{q}}{x^{2 q}} \varphi\left(a_{m} ; f\right)\left(\frac{a_{m}}{m}\right)^{q}\left(x\left(1-\frac{x}{a_{m}}\right)+\frac{a_{m}}{m}\right)^{q}+\frac{2 \vartheta|f(x+)-f(x-)|}{\sqrt{\frac{m x}{a_{m}}\left(1-\frac{x}{a_{m}}\right)}} .
$$

In order to show this it is necessary to prove that the right-hand sides of the inequalities mentioned in the hypotheses of the theorems tend to zero as $m \rightarrow \infty$. In view of (4) we have $n=\left[\sqrt{m / a_{m}}\right] \rightarrow \infty$ as $m \rightarrow \infty$. So, in Theorem 1 it is enough to consider only the term

Clearly,

$$
\wedge_{n}(x)=\sum_{j=1}^{n-1} \frac{Q_{j}\left(g_{x} ; H_{x}\left(j x d_{m}\right)\right)}{j^{3}}, \text { where } d_{m}=\sqrt{a_{m} / m} \text {. }
$$

$$
\begin{gathered}
\wedge_{n}(x)=\sum_{j=1}^{n-1} \frac{\vartheta_{1}\left(g_{x} ; H_{x}\left(j x d_{m}\right)\right)}{j^{2}} \leq 4 d_{m} \int_{d_{m}}^{n d_{m}} \frac{\Xi_{1}\left(g_{x} ; H_{x}(x t)\right)}{t^{2}} d t \\
\leq 4 d_{m} \int_{1}^{n+1} \square_{1}\left(g_{x} ; H_{x}\left(\frac{x}{s}\right)\right) d s \leq \frac{4}{n} \sum_{i=1}^{n} \vartheta_{1}\left(g_{x} ; H_{x}\left(\frac{x}{i}\right)\right) .
\end{gathered}
$$

Since the function $g_{x}$ is continuous at $x$ and $\mho_{1}\left(g_{x} ; H_{x}\left(\frac{x}{i}\right)\right)$ denotes the oscillation of $g_{x}$ on the interval $H_{x}\left(\frac{x}{i}\right)$, we have

and consequently,

$$
\lim _{i \rightarrow \infty} \mathbb{2}_{1}\left(g_{x} ; H_{x}\left(\frac{x}{i}\right)\right)=0
$$

$$
\lim _{n \rightarrow \infty} \wedge_{n}(x)=0
$$

As regards Theorem 3.2, it is easy to verify that in view of the continuity of $g_{x}$ at $x$,

$$
\lim _{n \rightarrow \infty} \frac{1}{n^{1+1 / p}} \sum_{i=1}^{n^{2}-1} \frac{1}{(\sqrt{i})^{1-1 / p}} V_{p}\left(g_{x} ; H_{x}\left(\frac{x}{\sqrt{i}}\right)\right)=0 .
$$

Thus we get the following approximation theorem.

Proof. Let $f \in B V_{l o c}^{p}[0, \infty), p \geq 1$. In view of (6) and the notation $d_{m}=\sqrt{a_{m} / m}, n=\left[\sqrt{m / a_{m}}\right]$, we have

$$
\begin{aligned}
& \sum_{j=1}^{n-1} \frac{a_{j}\left(g_{x} ; H_{x}\left(j x d_{m}\right)\right)}{j^{3}} \leq \sum_{j=1}^{n-1} \frac{V_{p}\left(g_{x} ; H_{x}\left(j x d_{m}\right)\right)}{j^{2+1 / p}} \leq\left(2 d_{m}\right)^{2+1 / p} \int_{d_{m}}^{n d_{m}} \frac{V_{p}\left(g_{x} ; H_{x}(x t)\right)}{t^{2+1 / p}} d t \\
& \quad \leq\left(\frac{2}{n}\right)^{2+1 / p} \int_{1}^{(n+1)^{2}} \frac{V_{p}\left(g_{x} ; H_{x}(x / \sqrt{s})\right)}{(\sqrt{s})^{2+1 / p}} d s \leq\left(\frac{2}{n}\right)^{2+1 / p} \sum_{i=1}^{(n+1)^{2}-1} \frac{V_{p}\left(g_{x} ; H_{x}(x / \sqrt{i})\right)}{(\sqrt{i})^{2+1 / p}}
\end{aligned}
$$

and

moreover,

$$
\frac{\Xi_{n}\left(g_{x} ; H_{x}(x)\right)}{n^{2}} \leq \frac{V_{p}\left(g_{x} ; H_{x}(x)\right)}{n^{2+1 / p}}
$$

$$
\text { 国 }\left(g_{x} ; H_{x}\left(x \sqrt{a_{m} / m}\right)\right) \leq V_{p}\left(g_{x} ; H_{x}\left(x \sqrt{a_{m} / m}\right)\right)
$$

The estimation given in Theorem 3.2 now immediately follows from Theorem 3.1.

Corollary. Suppose that $f \in X_{l o c}[0, \infty)$ (in particular, $f \in B V_{l o c}^{p}[0, \infty), p \geq 1$ ) and that there exists a positive integer $q$ such that

$$
\lim _{m \rightarrow \infty}\left(\frac{a_{m}}{m}\right)^{q} \varphi\left(a_{m} ; f\right)=0 .
$$

Then at every point $x \in[0, \infty)$ at which the limits $f(x+), f(x-)$ exist we have

$$
\lim _{m \rightarrow \infty}\left(D_{m, \vartheta} f\right)(x)=\frac{f(x+)+\vartheta f(x-)}{\vartheta+1}
$$

Obviously, the above relations hold true for every measurable function $f$ bounded on $[0, \infty)$, in particular for every function $f$ of bounded $p$-th power variation $(p \geq 1)$ on the whole interval $[0, \infty)$. 


\section{References}

[1]. Agratini O., 2001, “An approximation process of Kantorovich type”, Math. Notes (Miskolc), No. 1, pp. 3-10.

[2]. Albrycht J. and Radecki J., 1960, "On a generalization of the theorem of voronovskaya", Zeszyty Nauk. Uniw. Mickiewicza No. 25, pp. 3-7.

[3]. Aniol G. and Pych-Taberska P., 2001, "Some properties of the Bezier-Durremeyer type operators", Comment. Math. Prece Mat. 41,pp.1-11.

[4]. Bezier,P. 1972, "Numerical Control Mathematics and Applications", Wiley, London.

[5]. Butzer P. L. and Karsli H., 2009, "Voronovskaya-type theorems for derivatives of the Bernstein- Chlodovsky polynomials and the Szasz-Mirakyan operator", Comment Math. 49, No.1, pp. 33-57.

[6]. Chanturia Z. A., 1974, "The moduli of variation of a function and its applications in the theory of Fourier series", (Russian) Dokl. Akad. Nauk SSSR 214(1974), 63-66; English transl: Sav. Math. Dokl.15, pp. 67-71.

[7]. Durrmeyer J. L., 1967, "Une formule d'inverse de la transformee de Laplace-applications a la theorie des moments", These de 3e cycle, Faculte des Sciences de l'Universite de Paris.

[8]. Derriennic M. M., 1981, "Sur I'approximation de fonctions integrables sur [0,1] par des polynomes de Bernstein modifies", J. Approx. Theory 31, No. 4, pp. 325-343.

[9]. Govil N. K. and Gupta V., 2006, "Simultaneous approximation for the Bezier variant of Baskakov-beta operators", Math. Comput. Modelling 44, No. 11-12, pp. 1153-1159.

[10]. Guo S. S., 1987, “On the rate of convergence of the Durrmeyer operator for functions of bounded variation”, J. Approx. Theory 51, No.2, pp. 183-192.

[11]. Gupta V., 2005, “An estimate on the convergence of Baskakov-Bezier operators”, J. Math. Anal. Appl. 312, No. 1, pp. $280-288$.

[12]. Gupta V., 2004, "Rate of convergence of Durrmeyer type Baskakov-Bezier operators for locally bounded functions", Turkish J. Math. 28, No. 3, pp. 271-280.

[13]. Heilmann M., 1989, "Direct and converse results for operators Baskakov-Durrmeyer type", Approx. Theory Appl. 5 No. 1, pp. 105-127.

[14]. Ibikli E. and Karsli H., 2005, "Rate of convergence of Chlodowsky type Durrmeyer operators", JIPAM. J. Inequal. Pure Appl. Math. 6, No. 4, Article 106, 12 pp. (electronic).

[15]. Karsli H., 2007, "Order of convergence of Chlodovsky type Durrmeyer operators for functions with derivatives of bounded variation", Indian. J. Pure Appl. Math. 38, No. 5, pp. 353-363.

[16]. Karsli H. and Ibikli E., 2008, "Convergence rate of a new Bezier variant of Chlodowsky operators to bounded variation functions", J. Compute. Appl. Math. 212, No. 2, pp. 431-443.

[17]. Karsli H. and Ibikli E., 2007, "Rate of convergence of Chlodowsky-type Bernstein operators for functions of bounded variation", Numer. Funct. Anal. Optim. 28, No. 3-4, pp. 367-378.

[18]. Lagrange M. R., 1965, “Sur les oscillations d'order superieur d'une function numerique”, Ann. Sci. Ecole Norm. Sup. (3) 82, pp. 101-130.

[19]. Lorentz G. G., 1953, "Bernstein polynomials. Mathematical Expositions”, University of Toronto Press, Toronto.

[20]. Pych-Taberska P., 2003, "Some properties of the Bezier-Kantrovich type operators”, J. Approx. Theory 123, No. 2, pp. 256-269.

[21]. Srivastava H. M. and Gupta V., 2005, "Rate of convergence for the Bezier variant of the Bleimann-Butzer-Hahn operators", Appl. Math. Lett. 18, No. 8, pp. 849-857.

[22]. Zeng X. M. and Chen W., 2000, "On the rate of convergence of the generalized Durrmeyer type operators for functions of bounded variation", J. Approx. Theory 102, No. 1, pp. 1-12.

[23]. Zeng X. M. and Piriou A., 1998, "On the rate of convergence of two Bernstein-Bezier type operators for bounded variation functions", J. Approx. Theory 95, No. 3, pp. 369-387. 